11

\title{
Рекуперация в сверхмощных черенковских генераторах с неоднородным магнитным полем
}

\author{
(C) А.А. Кураев, ${ }_{1}^{1}$ A.О. Рак, ${ }^{1}$ C.A. Куркин, ${ }^{2,3,}$ А.А. Короновский, ${ }^{2,3}$ А.А. Бадарин, ${ }^{2,3}$ A.Е. Храмов ${ }^{2,3}$ \\ ${ }^{1}$ Белорусский государственный университет информатики и радиоэлектроники, \\ 220013 Минск, Беларуссия \\ ${ }^{2}$ Саратовский госуниверситет им. Н.Г. Чернышевского, \\ 410012 Саратов, Россия \\ ${ }^{3}$ Саратовский государственный технический университет им. Ю.А. Гагарина, \\ 410058 Саратов, Россия \\ ฯ e-mail: KurkinSA@gmail.com
}

(Поступило в Редакцию 30 сентября 2015 г. В окончательной редакции 22 марта 2016 г.)

Рассмотрен эффективный способ повышения полного коэффициента полезного действия (КПД) релятивистских черенковских генераторов - рекуперация. Установлено, что для генератора с КПД менее $15 \%$ использование рекуперации позволило получить прирост КПД более 50\% (с 12\% до 68\%). В генераторах с изначально высоким КПД (более 50\%) применение рекуперации может оказаться неэффективным.

\section{Введение}

В последние годы в различных областях науки и техники большое распространение получили мощные генераторы и усилители СВЧ-излучения [1-5]. Мощное СВЧ-излучение используется для радиолокации, радиопротиводействия, ускорения ионов, передачи и обработки информации в системах дальней связи, при изучении взаимодействия излучения с веществом, в биологических исследованиях и т.д. [1-7]. К одному из наиболее обширных классов мощных приборов микроволновой электроники относятся источники излучения, принцип действия которых основан на продольном взаимодействии электронного потока с электромагнитным полем в периодических электродинамических структурах, имеющих положительную или отрицательную дисперсию основной волны. Наибольшее усиление и устойчивая генерация в устройствах данного класса наблюдаются при реализации синхронизма потока и поля на частотах вблизи границ полосы прозрачности волноведущих систем. В этих частотных диапазонах все периодические системы проявляют резонансные свойства, в них одновременно могут возбуждаться прямые и обратные волны. В сверхмощной электронике, где обычно применяются релятивистские сильноточные электронные пучки, перспективными оказались черенковские и дифракционные генераторы, использующие резонансные периодические структуры с положительной дисперсией основной волны и поперечными размерами, значительно превышающими длину волны излучения. Создание сильноточных ускорителей электронов с токами $1-35 \mathrm{kA}$ при напряжении 0.3-2 MV позволяет реализовать черенковские генераторы СВЧ диапазона с электродинамической системой в виде отрезка периодического гофрированного полого волновода, имеющие выходную мощность $1-30 \mathrm{GW}$ в сантиметровом и миллиметровом диапазонах при КПД $10-50 \%$. Такие результаты до- стигнуты с использованием простейших математических моделей, справедливых для неглубокой периодической гофрировки волновода. Повышение КПД и улучшение выходных характеристик генераторов возможно на основе оптимизации всех параметров. Например, использование оптимального нерегулярного профиля в релятивистский черенковских генераторах (РЧГ) позволяет получать КПД порядка 40-50\% [5-8], а дальнейшее повышение эффективности прибора возможно за счет использования рекуперации.

\section{Описание модели}

В настоящей работе рассматривались РЧГ, схема которых представлена на рис. 1. Эти РЧГ и электростатическое поле в них обладают аксиальной симметрией, задача решалась в цилиндрических координатах, а поле рассчитывалось в двумерном приближении. Электростатическое поле в системе рассчитывалось методом конечных элементов. Уравнение Лапласа в цилиндрических координатах имеет следующий вид:

$$
\begin{aligned}
\nabla^{2} \varphi=\operatorname{div} g \operatorname{rad} \varphi & =\frac{1}{r} \frac{\partial}{\partial r}\left(r \frac{\partial \varphi}{\partial r}\right)+\frac{\partial^{2} \varphi}{\partial z^{2}}=0, \\
\mathbf{E} & =-\operatorname{grad} \varphi,
\end{aligned}
$$

где $r, z-$ поперечная и продольная координаты, $\varphi-$ потенциал электрического поля, $\mathbf{E}-$ вектор напряженности электрического поля.

Для осаждения электронов на коллектор использовалось неоднородное магнитное поле: $B_{0}-$ магнитное поле вдоль оси, которое задается следующим выражением:

$$
B_{0}(z)=B_{0 \max }+\left(B_{0 \min }-B_{0 \max }\right) \sin ^{2}\left(\frac{\pi z}{2 L_{m}}\right) .
$$

Здесь $L_{m}-$ область изменения магнитного поля. 


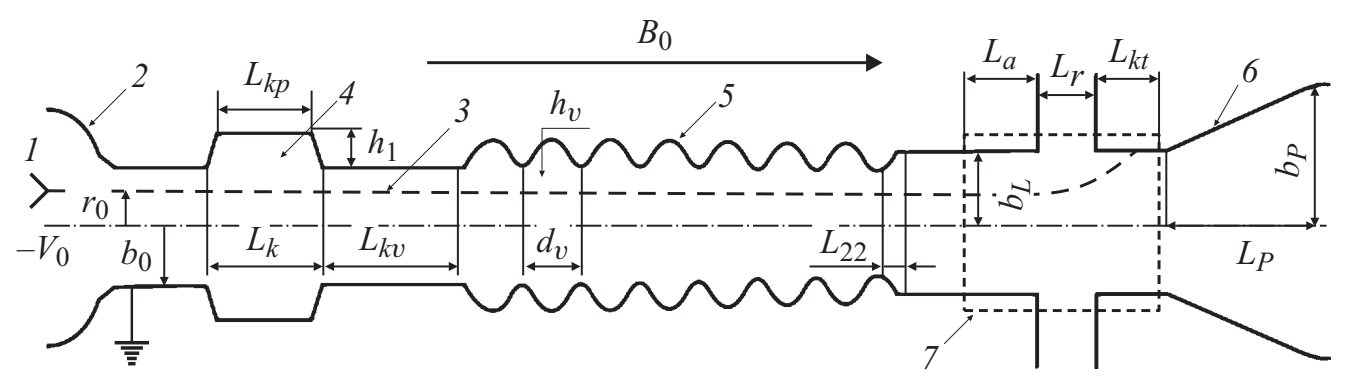

Рис. 1. Схема черенковского генератора. 1,2 - катод и анод, формирующие трубчатый электронный поток, $3,4-$ модулирующая канавка, 5 - периодическая замедляющая гребенка, 6 - выходной рупор, 7 - область рекуперации.

Выражение составляющих вектора $\mathbf{B}$ через $B_{0}(z)$ :

$$
\left\{\begin{array}{l}
B_{r}=-\frac{1}{2} r_{l} \frac{\partial B_{0}(z)}{\partial z}+\frac{1}{16} r_{l}^{3} \frac{\partial^{3} B_{0}(z)}{\partial z^{3}} \\
B_{z}=B_{0}(z)-\frac{1}{4} r_{l}^{2} \frac{\partial^{2} B_{0}(z)}{\partial z^{2}} .
\end{array}\right.
$$

Уравнение движения релятивистского электрона [9]

$$
\frac{d \mathbf{v}}{d t}=\frac{e}{m_{0}} \sqrt{1-\frac{v^{2}}{c^{2}}}\left(\mathbf{E}+[\mathbf{v}, \mathbf{B}]-\frac{\mathbf{v}}{c^{2}}(\mathbf{v}, \mathbf{E})\right),
$$

где $e, m_{0}$ - заряд и масса покоя электрона, $c$ скорость света в вакууме.

Для численного интегрирования уравнений движения использовался специальный алгоритм интегрирования дифференциальных уравнений второго порядка [10]:

Предсказание

$$
\begin{gathered}
x_{t+\Delta t}=x_{t}+v_{t} \Delta t+\frac{1}{8}\left(5 a_{t}-a_{t-\Delta t}\right) \Delta t^{2}+O\left(\Delta t^{3}\right), \\
v_{t+\Delta t}=v_{t}+\frac{1}{2}\left(3 a_{t}-a_{t-\Delta t}\right) \Delta t+O\left(\Delta t^{3}\right) .
\end{gathered}
$$

Коррекция

$$
\begin{aligned}
& x_{t+\Delta t}=x_{t}+v_{t} \Delta t+\frac{1}{8}\left(a_{t+\Delta t}+3 a_{t}\right) \Delta t^{2}+O\left(\Delta t^{3}\right), \\
& v_{t+\Delta t}=v_{t}+\frac{1}{8}\left(3 a_{t+\Delta t}+6 a_{t}-a_{t-\Delta t}\right) \Delta t+O\left(\Delta t^{3}\right),
\end{aligned}
$$

где $x(t)$ - координата частицы, $v(t), a(t)$ - соответственно ее скорость и ускорение, $\Delta t-$ шаг интегрирования по времени.

В качестве начальных условий для электронов использовались значения положения и скоростей частиц, полученные при моделировании черенковских генераторов [5-8].

Полный КПД определялся через потерю энергии электронным потоком

$$
\begin{gathered}
\eta=\frac{1}{N} \sum_{l=1}^{N} \frac{\gamma_{0}-\gamma_{l}}{\gamma_{0}-1}, \\
\gamma=\frac{1}{\sqrt{1-\left(\frac{\mathbf{v}}{c}\right)^{2}}},
\end{gathered}
$$

где $N-$ количество частиц, $l-$ номер частицы, $\gamma_{0}, \gamma-$ релятивистский фактор частиц на входе и выходе прибора соответственно.

\section{Результаты моделирования}

С использованием математической теории, развитой в [5-8], были найдены оптимальные варианты РЧГ с различными параметрами и значениями КПД. Полученные варианты использовались для оценки эффективности рекуперации в приборах данного типа.

Параметры рассматриваемых вариантов.

Вариант 1. 3-сантиметровый одноволновой генератор. Рабочая частота $f=10 \mathrm{GHz}$, выбранная опорная частота $\omega_{0}$ соответствует $\lambda_{0}=3 \mathrm{~cm}$. Напряжение пучка $V_{0}=520 \mathrm{kV}\left(\beta_{0}=0.869\right)$, ток $I_{0}=2500 \mathrm{~A}$, величина фокусирующего магнитного поля $B_{0}=0.357 \mathrm{~T}$. Регулярный гофрированный участок имеет $n_{v}=10$ периодов, $d_{v}=2.62, h_{v}=1.45, b_{0}=3.75(1.26 \mathrm{~cm}, 0.69 \mathrm{~cm}$, $1.79 \mathrm{~cm})$. Ширина, высота модулирующей канавки и ее расстояние от начала гребенки $L_{1}=2.22, h_{1}=1.092$, $L_{1 v}=5.79(1.06 \mathrm{~cm}, 0.52 \mathrm{~cm}, 2.76 \mathrm{~cm}), \Delta_{p c}=0.8$. Радиус пучка $r_{0}=3.1(1.48 \mathrm{~cm})$. Количество слоев электронов - 2, электронов на слой - 8. Достигнутый КПД составил $23.5 \%$.

Вариант 2. 3-сантиметровый одноволновой генератор. Рабочая частота $f=10 \mathrm{GHz}$, выбранная опорная частота $\omega_{0}$ соответствует $\lambda_{0}=3 \mathrm{~cm}$. Напряжение пучка $V_{0}=228 \mathrm{kV}\left(\beta_{0}=0.723\right)$, ток $I_{0}=1600$ A, величина фокусирующего магнитного поля $B_{0}=1.79 \mathrm{~T}$. Регулярный гофрированный участок имеет $n_{v}=10$ периодов, $d_{v}=3$, $h_{v}=1.58, b_{0}=5(1.43,0.754,2.39 \mathrm{~cm})$. Ширина, высота модулирующей канавки и ее расстояние от начала гребенки $L_{1}=6, h_{1}=1.59, L_{1 v}=7.15(2.86,0.759,3.41 \mathrm{~cm})$, $\Delta_{p}=0.8$. Радиус пучка $r_{0}=4.2(2 \mathrm{~cm})$. Количество слоев электронов - 2, электронов на слой -8 . Достигнутый КПД составил $39.6 \%$.

Вариант 3. 6-сантиметровый одноволновой генератор. Рабочая частота $f=5 \mathrm{GHz}$, выбранная опорная частота $\omega_{0}$ соответствует $\lambda_{0}=6 \mathrm{~cm}$. Напряжение пучка $V_{0}=291 \mathrm{kV}\left(\beta_{0}=0.772\right)$, ток $I_{0}=823 \mathrm{~A}$, величина фокусирующего магнитного поля $B_{0}=0.179$ Т. Регулярный гофрированный участок имеет $n_{v}=7$ периодов, $d_{v}=2.35, h_{v}=1.31, b_{0}=2.8(2.24 \mathrm{~cm}, 1.31 \mathrm{~cm}, 2.67 \mathrm{~cm})$. Ширина, высота модулирующей канавки и ее расстояние от начала гребенки $L_{1}=1.5, h_{1}=1.38, L_{1 v}=4.63(1.43$, $1.32,4.42 \mathrm{~cm}), \Delta_{p}=0.8$. Радиус пучка $r_{0}=2(1.9 \mathrm{~cm})$. Ко- 
Таблица 1. Параметры рассматриваемых РЧГ с одиночной модулирующей канавкой

\begin{tabular}{|c|c|c|c|c|c|c|c|c|c|c|c|c|c|c|c|c|}
\hline № & $\begin{array}{c}f, \\
\mathrm{GHz}\end{array}$ & $\begin{array}{l}V_{0} \\
\mathrm{kV}\end{array}$ & $\beta_{0}$ & $\begin{array}{c}I_{0} \\
\mathrm{~A}\end{array}$ & $\begin{array}{c}B_{0} \\
\mathrm{~T}\end{array}$ & $n_{v}$ & $\begin{array}{l}d_{v} \\
\mathrm{~cm}\end{array}$ & $\begin{array}{l}h_{\nu} \\
\mathrm{cm}\end{array}$ & $\begin{array}{l}b_{0}, \\
\mathrm{~cm}\end{array}$ & $\begin{array}{l}L_{1}, \\
\mathrm{~cm}\end{array}$ & $\begin{array}{l}h_{1}, \\
\mathrm{~cm}\end{array}$ & $\begin{array}{l}L_{1 v}, \\
\mathrm{~cm}\end{array}$ & $\Delta_{p}$ & cfm & $\begin{array}{c}\text { Количество } \\
\text { слоев электронов / } \\
\text { электронов на слой }\end{array}$ & $\begin{array}{c}\text { КПД, } \\
\%\end{array}$ \\
\hline 1 & 10 & 520 & .869 & 2500 & 0.357 & 10 & 1.26 & 0.69 & 1.79 & 1.06 & 0.52 & 2.76 & 0.8 & 1.48 & $2 / 8$ & 23.5 \\
\hline 2 & 10 & 228 & 0.723 & 1600 & 1.79 & 10 & 1.43 & 0.754 & 2.39 & 2.86 & 0.759 & 3.41 & 0.8 & 2 & $2 / 8$ & 39.6 \\
\hline 3 & 5 & 291 & 0.772 & 823 & 0.179 & 7 & 2.24 & 1.31 & 2.67 & 1.43 & 1.32 & 4.42 & 0.8 & 1.9 & $2 / 8$ & 52.3 \\
\hline
\end{tabular}

Таблица 2. Параметры рассматриваемых РЧГ с двойной модулирующей канавкой

\begin{tabular}{|c|c|c|c|c|c|c|c|c|c|c|c|c|c|c|c|c|}
\hline № & $\begin{array}{c}f, \\
\mathrm{GHz}\end{array}$ & $\begin{array}{l}V_{0}, \\
\mathrm{kV}\end{array}$ & $\beta_{0}$ & $\begin{array}{c}I_{0} \\
\mathrm{~A}\end{array}$ & $\begin{array}{c}B_{0}, \\
\mathrm{~T}\end{array}$ & $n_{v}$ & $\begin{array}{l}d_{v} \\
\mathrm{~cm}\end{array}$ & $\begin{array}{l}h_{\nu} \\
\mathrm{cm}\end{array}$ & $\begin{array}{l}b_{0}, \\
\mathrm{~cm}\end{array}$ & $\begin{array}{c}L_{1}=\underset{12}{L_{12}}=L_{2},\end{array}$ & $\begin{array}{l}h_{1}, \\
\mathrm{~cm}\end{array}$ & $\begin{array}{l}L_{2 v}, \\
\mathrm{~cm}\end{array}$ & $\Delta_{p}$ & $\begin{array}{l}r_{0}, \\
\mathrm{~cm}\end{array}$ & $\begin{array}{c}\text { Количество } \\
\text { слоев электронов / } \\
\text { электронов на слой }\end{array}$ & $\begin{array}{c}\text { КПД, } \\
\text { \% }\end{array}$ \\
\hline 4 & 10 & 323 & 0.84 & 3000 & 0.35 & 20 & 0.61 & 0.68 & 3.77 & 2.2 & 0.96 & 0.53 & 0.8 & 3.3 & $3 / 8$ & 42 \\
\hline 5 & 10 & 323 & 0.84 & 3000 & 0.35 & 20 & 0.605 & 0.7 & 3.77 & 2.23 & 0.96 & 0.38 & 0.8 & 3.3 & $2 / 8$ & 46 \\
\hline 6 & 10 & 323 & 0.84 & 2000 & 0.35 & 20 & 0.6 & 0.7 & 3.77 & 2.3 & 0.96 & 0.38 & 0.8 & 3.3 & $2 / 8$ & 12 \\
\hline 7 & 10 & 323 & 0.84 & 2000 & 0.35 & 20 & 0.612 & 0.69 & 3.77 & 2.23 & 0.96 & 0.38 & 0.8 & 3.3 & $2 / 32$ & 42.8 \\
\hline 8 & 10 & 323 & 0.84 & 1750 & 0.35 & 20 & 0.612 & 0.69 & 3.77 & 2.23 & 0.96 & 0.38 & 0.8 & 3.3 & $2 / 32$ & 13.14 \\
\hline
\end{tabular}

личество слоев электронов - 2, электронов на слой 8. Выбор относительно сильного фокусирующего поля позволил повысить электронный КПД до $52.3 \%$ за счет компенсации динамического расслоения.

Вариант 4. 3-сантиметровый двуволновой генератор. Рабочая частота $f=10 \mathrm{GHz}$, выбранная опорная частота $\omega_{0}$ соответствует $\lambda_{0}=3 \mathrm{~cm}$. Напряжение пучка $V_{0}=323 \mathrm{kV}\left(\beta_{0}=0.84\right)$, ток $I_{0}=3000 \mathrm{~A}$, величина фокусирующего магнитного поля $B_{0}=0.35 \mathrm{~T}$. Регулярный гофрированный участок имеет $n_{v}=20$ периодов, $d_{v}=1.28, h_{v}=1.43, b_{0}=7.9(0.61,0.68,3.77 \mathrm{~cm})$. Ширина, высота двойной модулирующей канавки и ее расстояние от начала гребенки $L_{1}=L_{12}=L_{2}=4.6$, $h_{1,2}=2, L_{2 v}=1.12(2.2,0.96,0.53 \mathrm{~cm}), \Delta_{p}=0.8$. Радиус пучка $r_{0}=6.9(3.3 \mathrm{~cm})$. Количество слоев электронов 3 , электронов на слой - 8. Достигнутый КПД составил $42 \%$.

Вариант 5. 3-сантиметровый двуволновой генератор. Рабочая частота $f=10 \mathrm{GHz}$, выбранная опорная частота $\omega_{0}$ соответствует $\lambda_{0}=3 \mathrm{~cm}$. Напряжение пучка $V_{0}=323 \mathrm{kV}\left(\beta_{0}=0.84\right)$, ток $I_{0}=3000 \mathrm{~A}$, величина фокусирующего магнитного поля $B_{0}=0.35 \mathrm{~T}$. Регулярный гофрированный участок имеет $n_{v}=20$ периодов, $d_{v}=1.268, \quad h_{v}=1.46, \quad b_{0}=7.9 \quad(0.605,0.7,3.77 \mathrm{~cm})$. Ширина, высота двойной модулирующей канавки и ее расстояние от начала гребенки $L_{1}=L_{12}=L_{2}=4.67$, $h_{1,2}=2, L_{2 v}=0.79(2.23,0.96,0.38 \mathrm{~cm}), \Delta_{p}=0.8$. Радиус пучка $r_{0}=6.9(3.3 \mathrm{~cm})$. Количество слоев электронов 2 , электронов на слой - 8. Достигнутый КПД составил $46 \%$.

Вариант 6. 3-сантиметровый двухволновой генератор. Рабочая частота $f=10 \mathrm{GHz}$, выбранная опорная частота $\omega_{0}$ соответствует $\lambda_{0}=3 \mathrm{~cm}$. Напряжение пучка $V_{0}=323 \mathrm{kV}\left(\beta_{0}=0.84\right)$, ток $I_{0}=2000 \mathrm{~A}$, величина фокусирующего магнитного поля $B_{0}=0.35$ T. Регулярный гофрированный участок имеет $n_{v}=20$ периодов, $d_{v}=1.25, h_{v}=1.46, b_{0}=7.9(0.6,0.7,3.77 \mathrm{~cm})$. Ширина, высота двойной модулирующей канавки и ее расстояние от начала гребенки $L_{1}=L_{12}=L_{2}=4.67$, $h_{1,2}=2, L_{2 v}=0.79(2.23,0.96,0.38 \mathrm{~cm}), \Delta_{p}=0.8$. Радиус пучка $r_{0}=6.9(3.3 \mathrm{~cm})$. Количество слоев электронов 2 , электронов на слой - 8. Достигнутый КПД составил $12 \%$.

Вариант 7. 3-сантиметровый двухволновой генератор. Рабочая частота $f=10 \mathrm{GHz}$, выбранная опорная частота $\omega_{0}$ соответствует $\lambda_{0}=3 \mathrm{~cm}$. Напряжение пучка $V_{0}=323 \mathrm{kV}\left(\beta_{0}=0.84\right)$, ток $I_{0}=2000 \mathrm{~A}$, величина фокусирующего магнитного поля $B_{0}=0.35$ T. Регулярный гофрированный участок имеет $n_{v}=20$ периодов, $d_{v}=1.282, h_{v}=1.45, b_{0}=7.9(0.612,0.69,3.77 \mathrm{~cm})$. Ширина, высота двойной модулирующей канавки и ее расстояние от начала гребенки $L_{1}=L_{12}=L_{2}=4.67$, $h_{1,2}=2, L_{2 v}=0.79(2.23,0.96,0.38 \mathrm{~cm}), \Delta_{p}=0.8$. Радиус пучка $r_{0}=6.9(3.3 \mathrm{~cm})$. Количество слоев электронов 2 , электронов на слой - 32. Достигнутый КПД составил $42.8 \%$.

Вариант 8. 3-сантиметровый двуволновой генератор. Рабочая частота $f=10 \mathrm{GHz}$, выбранная опорная частота $\omega_{0}$ соответствует $\lambda_{0}=3 \mathrm{~cm}$. Напряжение пучка $V_{0}=323 \mathrm{kV}\left(\beta_{0}=0.84\right)$, ток $I_{0}=1750 \mathrm{~A}$, величина фокусирующего магнитного поля $B_{0}=0.35$ T. Регулярный гофрированный участок имеет $n_{v}=20$ периодов, $d_{v}=1.282, h_{v}=1.45, b_{0}=7.9(0.612,0.69,3.77 \mathrm{~cm})$. Ширина, высота двойной модулирующей канавки и ее расстояние от начала гребенки $L_{1}=L_{12}=L_{2}=4.67$, $h_{1,2}=2, L_{2 v}=0.79(2.23,0.96,0.38 \mathrm{~cm}), \Delta_{p}=0.8$. Радиус пучка $r_{0}=6.9(3.3 \mathrm{~cm})$. Количество слоев электронов 2 , электронов на слой - 32. Достигнутый КПД составил $13.14 \%$.

Параметры рассматриваемых вариантов генераторов и их характеристики приведены в табл. 1 и табл.2. 

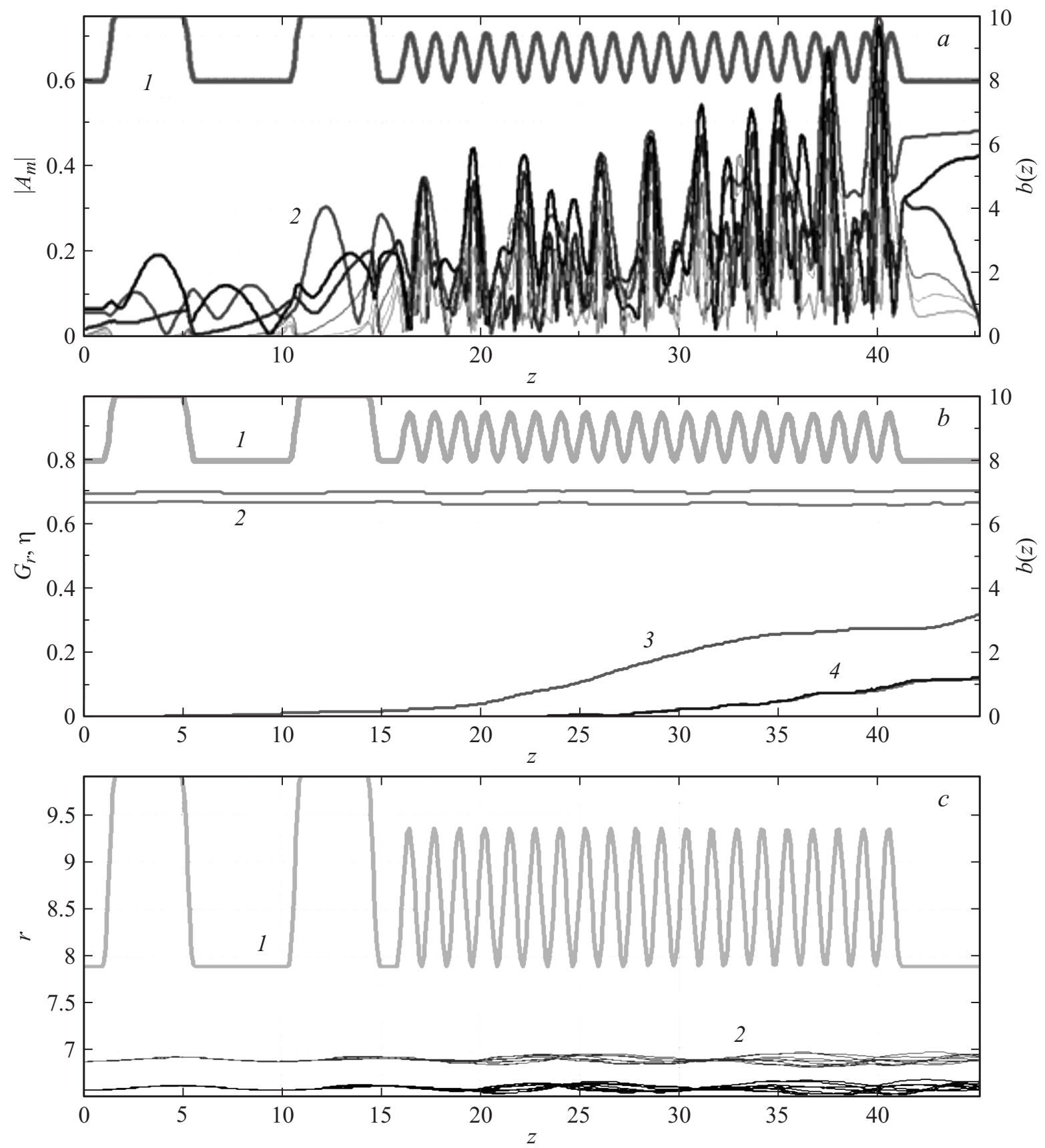

Рис. 2. Характеристики одного из оптимальных вариантов РЧГ (вариант 6): $a$ - профиль (1) и распределение амплитуд (2), $b-$ границы электронного потока (2), функция группировки (3), электронный и волновой КПД (4), $c$ - траектории электронов (2).

Приведем основные характеристики варианта 6 на рис. 2. Основную роль при моделировании рекуперации играет распределение электронов по скоростям. Данная характеристика для рассматриваемых генераторов представлена на рис. 3 .

На рис. 4 представлены траектории электронов двух радиальных слоев РЭП, движущихся в неоднородном магнитном поле при нулевом относительно анода потенциале коллектора.

Из рис. 4 (а также рис. 2,c) видно, что траектории электронов на выходе РЧГ (в данных условиях) носят сложный характер и могут быть определены только в результате численного моделирования. В связи с этим конфигурация рекуперационной системы должна оптимизироваться для каждого моделируемого прибора индивидуально.

Для поиска эффективных конфигураций оптимизировались следующие параметры: профиль неоднородного магнитного поля, геометрические размеры системы $\left(L_{a}\right.$, $\left.L_{r}, L_{k}\right)$, напряжение на коллекторе. При оптимизации выдерживалось условие осаждения всех электронов на коллектор (отсутствие отраженных и вышедших за об- 

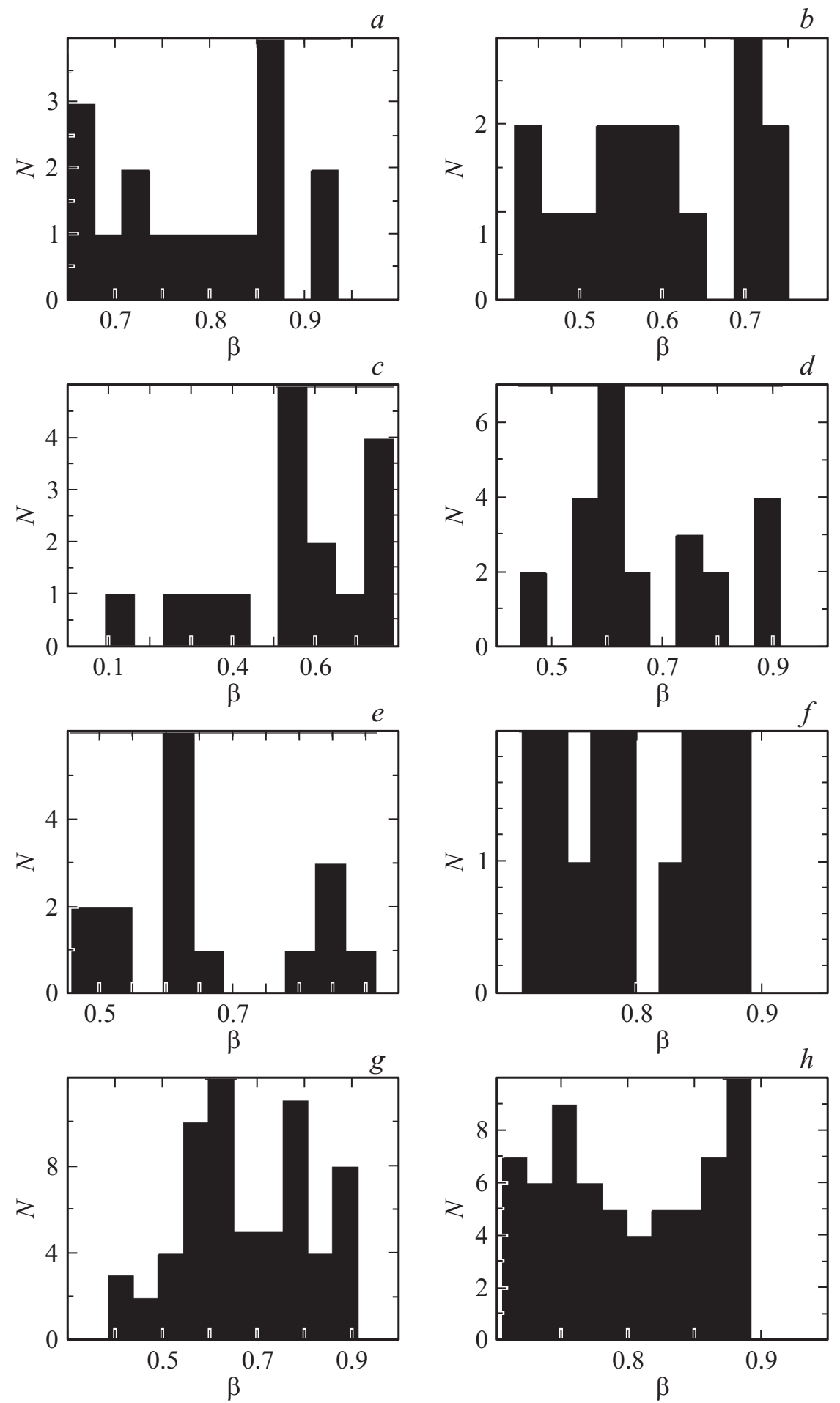

Рис. 3. Распределение электронов по скоростям для вариантов генераторов $1-8$.

ласть взаимодействия электронов). Полученные результаты приведены в табл. 3.

Параметры найденных оптимальных рекуперационных систем для рассматриваемых генераторов представлены в табл. 4.

Вариант 3 обладает весьма высоким КПД (52.3\%), в результате этого в приборе имеются электроны с низки- ми скоростями $(0.1 c-$ рис. $3, c)$, для предотвращения возврата таких электронов в область взаимодействия при введении рекуперации приходится менять режим работы генератора, ухудшая его электронный КПД. Для рассматриваемого варианта использование рекуперации не только не позволило повысить КПД, но даже привело к небольшому его снижению. 
Таблица 3. Результаты оптимизации РЧГ

\begin{tabular}{c|c|c|c}
\hline № & $\begin{array}{c}\text { Электронный } \\
\text { КПД генератора }\end{array}$ & Полный КПД & Прирост КПД \\
\hline 1 & 23.5 & 35.84 & 12.34 \\
2 & 39.6 & 64.14 & 24.54 \\
3 & 52.3 & 51.95 & -0.35 \\
4 & 42 & 55.74 & 13.74 \\
5 & 46 & 63.13 & 17.13 \\
6 & 12 & 68.9 & 56.9 \\
7 & 42.8 & 54.37 & 11.57 \\
8 & 13.14 & 68 & 54.86
\end{tabular}

Таблица 4. Параметры оптимальных рекуперационных систем для рассматриваемых генераторов

\begin{tabular}{c|c|c|c|l|c|l|l}
\hline № & $\begin{array}{c}L_{a}, \\
\mathrm{~cm}\end{array}$ & $\begin{array}{c}L_{r}, \\
\mathrm{~cm}\end{array}$ & $\begin{array}{c}L_{k t}, \\
\mathrm{~cm}\end{array}$ & $\begin{array}{l}U_{k}, \\
\mathrm{kV}\end{array}$ & $\begin{array}{c}L_{m}, \\
\mathrm{~cm}\end{array}$ & $\begin{array}{c}B_{0 \max }, \\
\mathrm{T}\end{array}$ & $\begin{array}{c}B_{0 \min }, \\
\mathrm{T}\end{array}$ \\
\hline 1 & 7.3 & 7.6 & 17.4 & -90 & 55 & 0.375 & 0 \\
2 & 2.9 & 1.1 & 3 & -55 & 6.7 & 1.7 & 1.1 \\
3 & 2 & 2 & 9.9 & -1.9 & 15 & 0.375 & 0 \\
4 & 5 & 8 & 10 & -60 & 55 & 0.375 & 0 \\
5 & 10 & 2.3 & 9.8 & -76.97 & 28.8 & 0.375 & 0.195 \\
6 & 10 & 1 & 10 & -246 & 23.4 & 0.375 & 0.231 \\
7 & 9.4 & 1 & 10 & -38 & 26 & 0.375 & 0.2175 \\
8 & 10 & 1 & 10 & -236 & 23.4 & 0.375 & 0.231
\end{tabular}

Для исследования рекуперации в генераторах с низким КПД был изменен вариант 5 следующим образом: понижен ток с 3000 до 2000 А и выполнена оптимизация периода гофра. В результате КПД был понижен с 46 до $12 \%$ (вариант 6). В полученном генераторе электроны обладаю высокими скоростями и относительно равномерным распределением, что позволило эффективно использовать рекуперацию и получить прирост КПД $56.9 \%$.

При моделировании РЧГ с использованием теории, развитой в [5-8] для получения достоверных результатов достаточно использовать небольшое количество крупных частиц (в рассмотренных вариантах в основном - 16), однако при моделировании рекуперации этого может оказаться недостаточно для учета сильно замедленных частиц. В вариантах 7, 8 увеличено число электронов до 64. Увеличение количества электронов отразилось на непрерывности распределения частиц по скоростям, однако, на эффективность использования рекуперации практически не повлияло.

Потенциал электростатического поля, профиль нерегулярного магнитного поля и траектории электронов для варианта 6 представлены на рис. 5, 6 и 7 соответственно.

Как видно из рис. 5 электростатическое поле в области взаимодействия представляет собой электронную линзу [11].

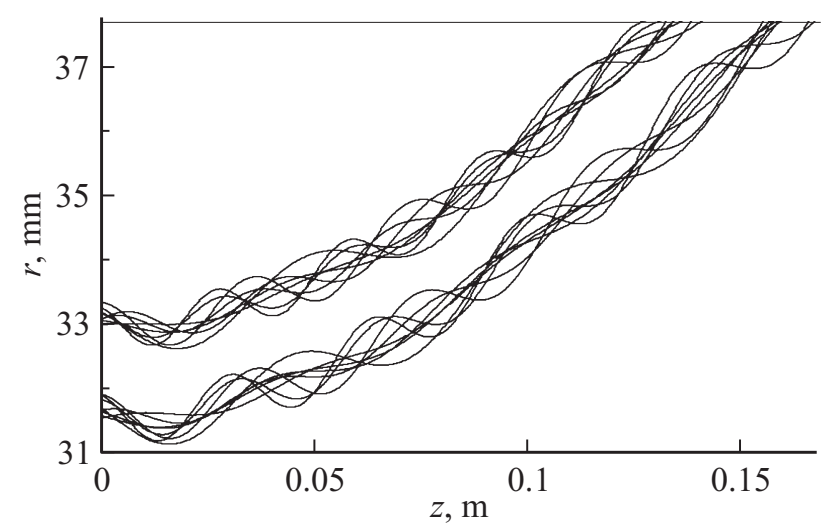

Рис. 4. Осаждение электронов при нулевом относительно анода потенциале коллектора.

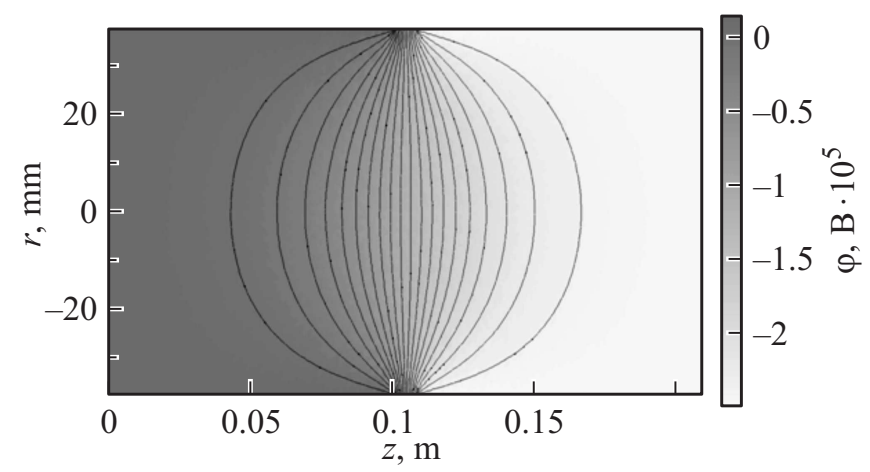

Рис. 5. Распределение потенциала электростатического поля для варианта 6.

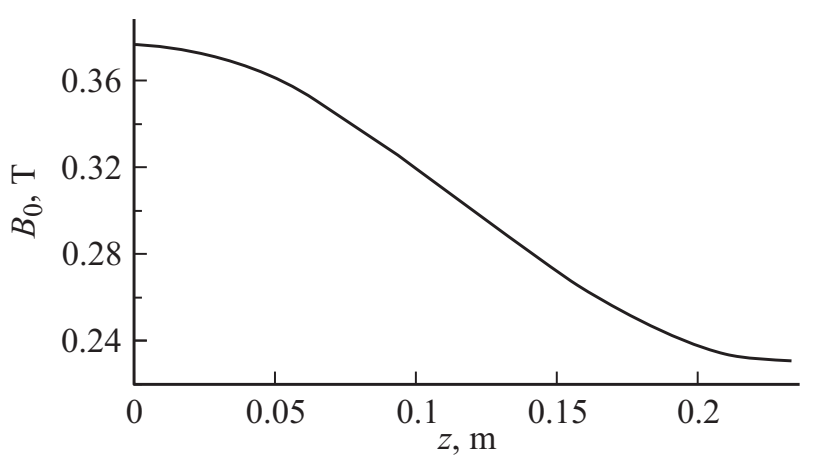

Рис. 6. Профиль неоднородного магнитного поля для варианта 6.

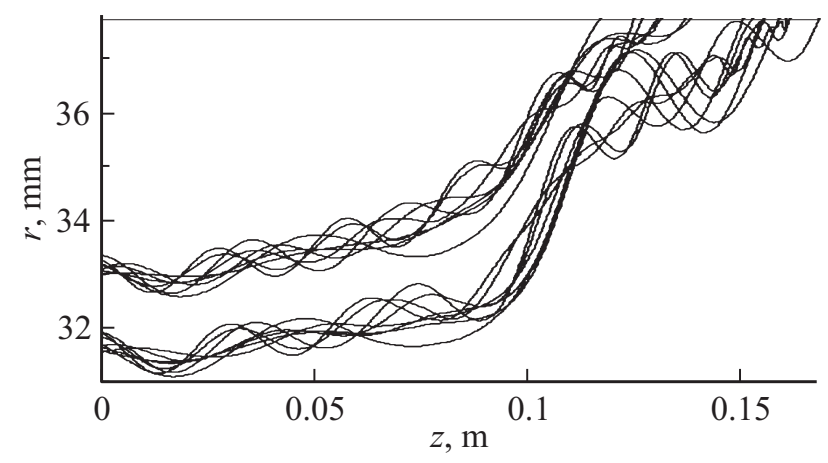

Рис. 7. Траектории электронов для варианта 6. 


\section{Заключение}

Анализ траекторий электронов и распределения их скоростей на выходе РЧГ показал, что для каждого отдельного генератора требуется индивидуальный расчет рекуперационной системы.

Для генератора с меньшим КПД и в результате этого менее замедленными и равномерно распределенными по скоростям на выходе прибора электронами использование рекуперации позволило получить больший прирост КПД - более $50 \%$. В генераторах с изначально высоким КПД (более 50\%) применение рекуперации может оказать неэффективным.

В результате оптимизации были получены конфигурации РЧГ с полным КПД, близким к 70\%.

Заметим, что эмиссия вторичных электронов с коллектора в настоящей работе не учитывалась, так как она оказывает меньшее влияние в схеме с рекуперацией, поскольку форма коллектора остается прежней (как без рекуперации), а энергия первичных электронов снижена.

Работа выполнена при поддержке грантов РФФИ (№ 15-52-04018, 15-32-20299), гранта президента РФ для молодых российских ученых - кандидатов наук (МК5426.2015.2) и Минобрнауки РФ (№ 3.59.2014/К).

\section{Список литературы}

[1] Трубецков Д.И., Храмов А.Е. Лекции по сверхвысокочастотной электронике для физиков. Т. 1. М.: Физматлит, 2003. $496 \mathrm{c}$.

[2] Трубеиков Д.И., Храмов А.Е. Лекции по сверхвысокочастотной электронике для физиков. Т. 2. М.: Физматлит, 2004. $648 \mathrm{c}$.

[3] Benford J., Swegle J.A., Schamiloglu E. High Power Microwaves. Taylor and Francis, CRC Press. 2016.

[4] Рухадзе А.А., Столбецов С.Д., Тараканов В.П. // РЭ. 1992. T. 37. № 3. C. 385

[5] Кураев А.А., Трубецков Д.И. Методы нелинейной динамики и теории хаоса в задачах электроники сверхвысоких частот. Т. 1. Стационарные процессы. М. : Физматлит, 2009. C. $167-274$.

[6] Кравченко В.Ф., Кураев А.А., Попкова Т.Л., Рак А.О. // Физические основы приборостроения. 2012. Т. 1. № 2. C. $41-64$.

[7] Кравченко В.Ф., Кураев А.А., ПопковаТ.Л., Рак А.О. // Физические основы приборостроения. 2013. Т. 2. № 1. C. $78-99$.

[8] Кураев А.А., Лущищкая И.В., Попкова Т.Л., Рак А.О., Синицын А.К. // Изв. вузов ПНД. 2008. Т. 16. № 3. C. $142-155$.

[9] Ландау Л.Д., Лифиии Е.М. Теория поля М.: Наука, 1988.

[10] Рак А.О. // Докл. БГУИР. 2012. Т. 67. № 5. С. 86.

[11] Арцимович Л.А., Лукьянов С.Ю. Движение заряженных частиц в электрических и магнитных полях. 2-е изд. М.: Наука, 1972. 